\title{
CORRELATION OF RISK FACTORS WITH HPE GRADING IN BREAST CANCER
}

Rudramurthy K. Gㅜㄹ Pradeep Kumar L², Avanthi E ${ }^{3}$, Ira Bharadwaj ${ }^{4}$

\section{HOW TO CITE THIS ARTICLE:}

Rudramurthy K. G, Pradeep Kumar L, Avanthi E, Ira Bharadwaj. “Correlation of Risk Factors with HPE Grading in Breast Cancer". Journal of Evolution of Medical and Dental Sciences 2014; Vol. 3, Issue 11, March 17;

Page: 2687-2692, DOI: $10.14260 /$ jemds/2014/2193

ABSTRACT: OBJECTIVE: To correlate risk factors for breast cancer with Histopathological grading. MATERIAL AND METHOD: A four year retrospective study was carried out from 2009-2012. 46 cases which were reported as breast cancer in due course were reviewed with histopathological (Scarff-Bloom-Richardson) grade of the tumor and familial, hormonal and acquired risk factors. The correlation of risk factors and the histopathological grade is done by using't' test. RESULTS: Among 46 cases of breast cancer, all were female. 16 cases were having familial risk factors, 12 cases were having hormonal risk factors and 12 cases were having acquired risk factors. 6 cases were having both familial and hormonal risk factors. In histopathological grading 12, 12, 22 cases were of grade I, II and III respectively. High grade was significantly correlating with familial and hormonal risk factors. There was a significant correlation with $\mathrm{p}$ value $<0.001$. CONCLUSION: Familial and hormonal risk factors are associated with high grade breast cancer than with that of acquired risk factors.

KEYWORDS: Breast Cancer, Risk factors, Scarff-Bloom Richardson grade.

INTRODUCTION: Breast carcinoma is the most common malignant tumor and leading cause of death in women, with more than 1, 00, 000 cases occurring worldwide annually. ${ }^{1}$ In United States, each year approximately 1, 00, 000 new cases are diagnosed and approximately 30, 000 patients die from the disease. There has been a sharp increase in the detection of breast carcinoma, largely due to the wide spread use of mammography. ${ }^{2}$ Most of these cases have a localized lesion with less than $2 \mathrm{~cm}$ in diameter. ${ }^{3}$

Many risk factors for the development of breast carcinoma has been established, whereas many other remain questionable. ${ }^{4}$ The most common risk factor is prolonged estrogen stimulation operating on a genetically susceptible individuals. ${ }^{5}$

Nottingham's modification of Bloom Richardson grading system is the most widely used microscopic grading for breast cancer. It is based on architectural features and mitotic activity. ${ }^{6}$ The system was largely applied for Infiltrating ductal carcinoma NOS, but can also be applied to special types of ductal carcinoma and Lobular carcinoma. ${ }^{7}$ Higher the histopathological grade better will be the response to the chemotherapy.

\section{AIMS AND OBJECTIVES:}

1. To evaluate the risk factors for breast cancer.

2. To correlate the risk factors with histopathological grade in breast cancer.

MATERIALS \& METHODS: A four year retrospective study was done from Jan 2009 - Dec 2012 in the department of Pathology, Karuna Medical College and Hospital, Kerala state, India. Patients who had undergone mastectomy for breast cancer and the patients with biopsy proven breast cancer were 
included in the study. Uncooperative patients and cases in which detailed history is not available were excluded from the study.

All the cases with breast cancer are reviewed. Detailed history with relevant risk factors was obtained from the case sheets. Patients are directly contacted wherever feasible and required.

\section{Risk factors are conveniently categorized into:}

A. Familial risk factors - Family history

- Genetic history

B. Hormonal risk factors -Early menarche

-Late menopause

-Late age of first child birth

-Nulliparity

-Exogenous Estrogen.

C. Acquired risk factor - Alcohol

- Fatty Diet

- Smoking

- Radiation

Histopathological section of all the cases are examined and graded according to Nottingham modified Bloom Richardson system ${ }^{8}$ into Grade I, II \& III. This System includes following parameters: Tubular formation, Nuclear Pleomorphism and Mitotic count.

Correlation of risk factors with that of histopathological grading is done with' $t$ ' test of statistical analysis.

RESULTS: A total of 46 cases were included in the study. All the cases were female. No male cases were there. Age of the patients were ranging from 30-87 years with a mean of 58.5 years (Table No.-1) Age of the youngest patient was 30 year and the oldest was 87 years.

Risk factors stratification is done based on the case sheets and on personal information.16 cases were having familial risk factors, 12 cases were having hormonal risk factors, 12 cases were having acquired risk factors and 6 cases were having both familial and hormonal risk factors (Table No 2).

There was no correlation with the age \& time of presentation to that of risk factors or HPE grade. Histopathogical grading was done with Nottingham modification of Bloom Richardson grading. 12 cases were showing grade I (Fig. 1), 12 cases were showing grade II (Fig. 2) and 22 cases were showing grade III (Fig. 3).

In the above observation it was obvious that the familial risk factor \& hormonal risk factors were associated with higher grade i.e. Grade III and Grade II respectively as compared to that of acquired factors with Grade I i.e. lower Grade.

On applying student' $\mathrm{t}$ ' test of correlation the $\mathrm{P}$ value of $<0.001$ is obtained. It suggests that there is a significant correlation between the risk factors and histopathological grade. Good correlation of familial and hormonal risk factors with higher Grade and acquired risk factors with lower Grade is obtained.

Thus we conclude that the patients with familial and hormonal risk factors can get a high grade breast cancer as compared to that of acquired risk factors. 
DISCUSSION: The incidence of breast cancer rises throughout a woman's lifetime, peak incidence is seen at the age of 75-80 years with declining slightly thereafter. The most important risk factor is gender; only $1 \%$ of male patients will get breast cancer. ${ }^{9}$ Common risk factors for women identified by epidemiologic studies have been combined into the Breast Cancer Risk Assessment Tool (BCRAT), which also includes information from the Contraceptive and Reproductive Experiences study, women with early menarche i.e. at less than 11 years of age have a $20 \%$ increased risk ${ }^{10}$ as compared to that of women who are more than 14 years of age at menarche. Late menopause will increase the risk. Women who have a first full-term pregnancy at the age younger than 20 years have half the risk in comparison to nulliparous women or women over the age of 35 at their first birth. ${ }^{11}$ It is hypothesized that the terminal differentiation of milk-producing luminal cells will occur in pregnancy, removing them from the potential pool of cancer precursors. The risk of getting breast cancer in an individual increases with the number of affected first-degree relatives (mother, sister, or daughter), especially if it occurs at young age. There is 1.2- 1.7 fold increase in the risk of getting breast cancer in case of postmenopausal hormone replacement therapy and adding progesterone increases the risk further. ${ }^{12}$ Exposure to any kind of radiation to the chest, whether due to cancer therapy, atomic bomb exposure, or nuclear accidents, increases the risk of breast cancer. Correlations between breast cancer risk and dietary intake of any specific type of food is not yet proved even with large studies. ${ }^{13}$ Moderate or heavy alcohol consumption will increases the risk. There is significant reduction in risk with breastfeeding. Cigarette smoking is not been clearly associated with breast cancer. ${ }^{14}$

The most commonly used grading system, the Nottingham Histological Score (also referred to as Scarff-Bloom-Richardson), combines nuclear grade, tubule formation and mitotic rate to classify invasive carcinomas into three groups those are highly correlated with survival. ${ }^{8}$

In the present study we have compared the risk factors with that of histopathological grading. With extensive search of literature no similar study has been found. We obtained a good correlation of familial and hormonal risk factors with that of high grade \& acquired factors with low grade.Carcinomas with high proliferation rates have a poorer prognosis but may respond better to chemotherapy. 8

Based on these findings we can conclude that the patient with familial and hormonal risk factors needs neo-adjuvant chemotherapy for better outcome. And the prognosis of the patients with familial and hormonal risk factors is poor in comparison to others. However a prospective study on a large group of patients is necessary as further supporting evidence.

CONCLUSION: Familial and hormonal risk factors are associated with higher histopathological grade as compared to that of other risk factors.

\section{REFERENCES:}

1. Parkin D M, Bray F, Ferlay J, Pisani P. Estimating the world cancer burden. Globocon 2000. Int J Cancer 2001; 94:153-6.

2. Sondik EJ. Breast cancer trends. Incidence, mortality and survival. Cancer 1994; 74:995-9.

3. Garfinkel L, Boring CC, Heath CW. Changing trends- An overview of breast cancer incidence and mortality. Cancer 1994; 74:222-7.

4. Armstrong K, Eisen A, Weber B. Assessing the risk of breast cancer. N Engl J Med 2000; 342:564-71. 


\section{ORIGINAL ARTICLE}

5. Moore DH, Moore DH II, Moore CT. Breast carcinoma etiological factors. Adv Cancer Research 1983; 40:189-253.

6. Bloom HJG, Richardson WW. Histological grading and prognosis in breast cancer. A study of 1409 cases if which 359 have been followed for 15 years. Br J Cancer 1957; 11:359-77.

7. Bane AL, Parkes R, Tjan S, O’Molley FP. Invasive lobular carcinoma: to grade or not to grade. Mod pathol 2003; 16:23.

8. Ellis IO, Elston CW. Histologic grade. In: O'Malley FP, Pinder SE, editor. Breast Pathology, Philadelphia: Elsevier; 2006. p. 225-33.

9. Russo J. Full-term pregnancy induces a specific genomic signature in the human Breast. Cancer Epidemiol Biomarkers Prev2008; 17:51.

10. Schedin P. Pregnancy-associated breast cancer and metastasis. Nat Rev Cancer 2006; 4:281.

11. Gail MH. Projecting individualized probabilities of developing breast cancer for White females who are being examined annually. J Natl Cancer Inst1989; 81:1879.

12. Gail MH. Projecting individualized absolute invasive breast cancer risk in African American women. J Natl Cancer Inst2007; 99:1782.

13. Hayanga AJ, Newman LA. Investigating the phenotypes and genotypes of breast cancer in women with African ancestry: the need for more genetic epidemiology. Surg Clin North Am 2007; 87:551.

14. John EM. Prevalence of pathogenic BRCA1 mutation carriers in 5 US racial/ethnic groups. JAMA 2007; 298:2910

\begin{tabular}{|c|c|}
\hline Age group & No. Of Cases \\
\hline $30-40$ & 10 \\
\hline $41-50$ & 16 \\
\hline $51-60$ & 10 \\
\hline $61-70$ & 07 \\
\hline$>71$ & 03 \\
\hline Total & $\mathbf{4 6}$ \\
\hline
\end{tabular}

Table 1: Distribution of cases according to age group 


\begin{tabular}{|c|c|}
\hline Risk Factors & No. of Cases \\
\hline Familial (F) & 16 \\
\hline -Family history & 10 \\
\hline -Genetic history & 06 \\
\hline Hormonal (H) & 12 \\
\hline -Early menarche & 04 \\
\hline -Late menopause & 03 \\
\hline -Late age of first child birth & 02 \\
\hline -Nulliparity & 02 \\
\hline -Exogenous Estrogen. & 01 \\
\hline Both F \& H & 06 \\
\hline Acquired & 12 \\
\hline - Alcohol & 04 \\
\hline - Fatty Diet & 02 \\
\hline - Smoking & 01 \\
\hline - Radiation & 05 \\
\hline Total & 46 \\
\hline
\end{tabular}

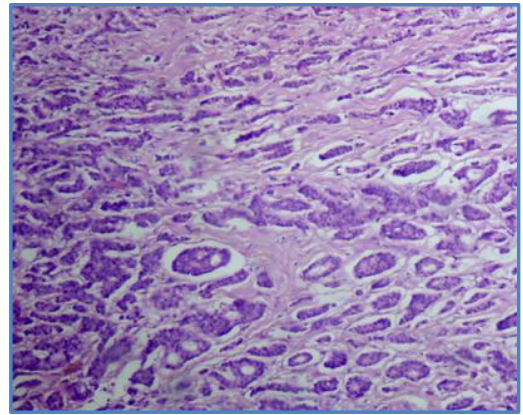

Fig. 1: Photomicrograph showing infiltrating ductal carcinoma grade I. (H\&E, 100X)

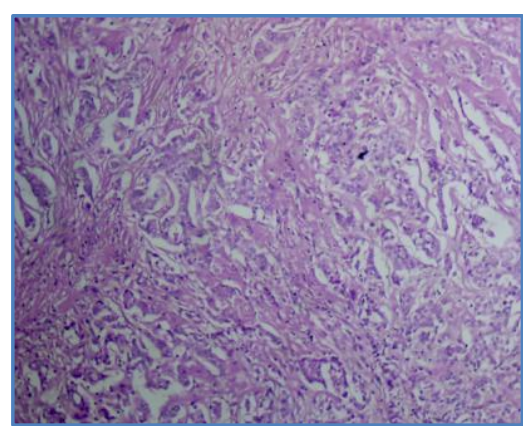

Fig. 2: Photomicrograph showing infiltrating ductal carcinoma grade II. (H\&E, 100X)

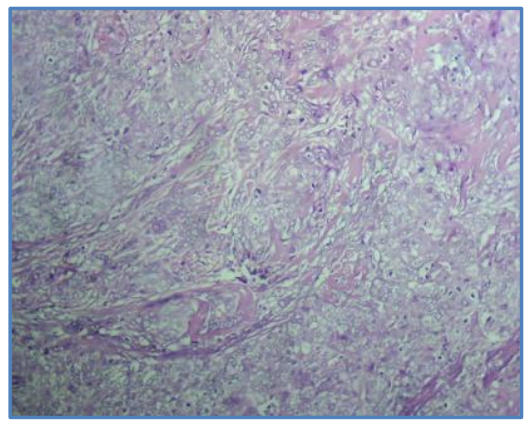

Fig. 3: Photomicrograph showing infiltrating ductal carcinoma grade III. (H\&E, 100X) 


\section{ORIGINAL ARTICLE}

\section{AUTHORS:}

1. Rudramurthy K. G.

2. Pradeep Kumar L.

3. Avanthi E.

4. Ira Bharadwaj

\section{PARTICULARS OF CONTRIBUTORS:}

1. Assistant Professor, Department of Microbiology, Karuna Medical College, Palakkad, Kerala.

2. Assistant Professor, Department of Pathology, Karuna Medical College, Palakkad, Kerala.

3. Post Graduate Tutor, Department of Pharmacology, JJMMC, Davangere.

4. Professor, Department of Pathology, Karuna Medical College, Palakkad, Kerala.

\section{NAME ADDRESS EMAIL ID OF THE} CORRESPONDING AUTHOR:

Dr. Pradeep Kumar L, Assistant Professor, Department of Pathology, Karuna Medical College, Vilayodi, Chittur, Palakkad, Kerala, India.

E-mail: pradeepnaik.1@gmail.com

Date of Submission: 14/02/2014. Date of Peer Review: 15/02/2014. Date of Acceptance: 22/02/2014. Date of Publishing: 11/03/2014. 